\title{
One-dimensional model for cooperative hydrogen motion in ferroelectric crystals
}

\author{
Authors: V. Hugo Schmidt
}

This is an Accepted Manuscript of an article published in Ferroelectrics in 1974, available online: http://www.tandfonline.com/10.1080/00150197408237994.

V.H. Schmidt, "One-dimensional model for cooperative hydrogen motion in ferroelectric crystals," Ferroelectrics 7, 199-200 (1974).

http://dx.doi.org/10.1080/00150197408237994

Made available through Montana State University's $\underline{\text { ScholarWorks }}$

scholarworks.montana.edu 


\title{
ONE-DIMENSIONAL MODEL FOR COOPERATIVE HYDROGEN MOTION IN FERROELECTRIC CRYSTALS ${ }^{\dagger}$
}

\author{
V. HUGO SCHMIDT \\ Department of Physics, Montana State University, Bozeman, Montana 59715, USA
}

(Received September 10, 1973)

\begin{abstract}
The static and dynamic behavior is examined for "Takagi groups" in double-minimum potential wells containing protons (or deuterons) coupled by harmonic forces. Predictions of this model are compared with observed isotope effects on domain wall mobility and dielectric relaxation in $\mathrm{KH}_{2} \mathrm{PO}_{4}$. The model predicts effects previously attributed to tunneling.
\end{abstract}

\section{INTRODUCTION}

Diffusion of Takagi $\mathrm{DPO}_{4}$ and $\mathrm{D}_{3} \mathrm{PO}_{4}$ groups explains most dynamical properties of $\mathrm{KD}_{2} \mathrm{PO}_{4}$ (DKDP), while cooperative tunneling has been invoked to explain the much more rapid dynamical response of $\mathrm{KH}_{2} \mathrm{PO}_{4}(\mathrm{KDP})$. As an alternative to the tunneling mechanism a onedimensional model for extended Takagi groups is developed, and used to predict certain dielectric phenomena in KDP and DKDP.

\section{MODEL FOR EXTENDED TAKAGI GROUPS}

The symmetric wells in Figure 1 indicate the effective potential per proton if all protons move in phase. The interproton forces are approximated by springs (not shown) of spring constant $k^{\prime}$ connecting neighboring protons. Given $k^{\prime}$ and an effective double well potential $U(x)=B x^{4}-A x^{2}$, the hydrogen positions can be found for the even and odd Takagi group configurations shown in Figure 1, as well as the respective potential energies $\epsilon_{1}-\epsilon_{\mu}$ and $\epsilon_{1}$ for these configurations.
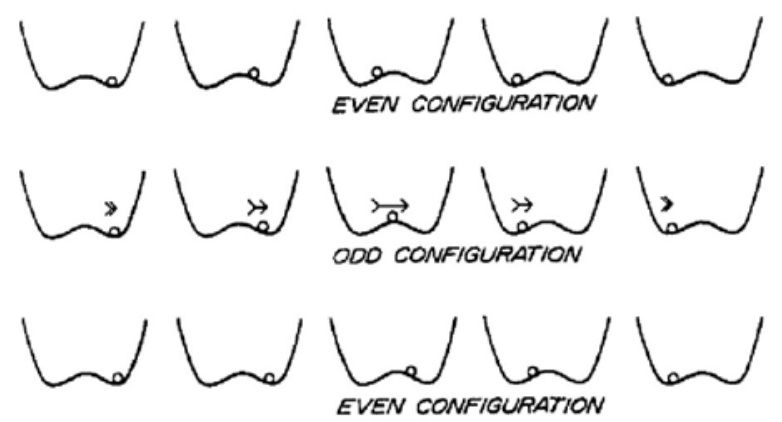

FIGURE I Takagi group transfer showing odd and even configurations. Arrows indicate proton displacements involved in moving the Takagi group one lattice space.

† This work supported by National Science Foundation.
The even configurations for six combinations of $A, B$, and $k^{\prime}$ are shown in Figure 2, toge ther with the energies $\epsilon_{1}$ and $\epsilon_{\mu}$. Each $A, B$ combination gives minima at the measured ${ }^{2}$ off-center displacements, taken as $x_{0}=0.200 \mathrm{~A}^{\circ}$ for DKDP and $0.164 \mathrm{~A}^{\circ}$ for KDP. For the top two rows of Figure 2, it was further assumed
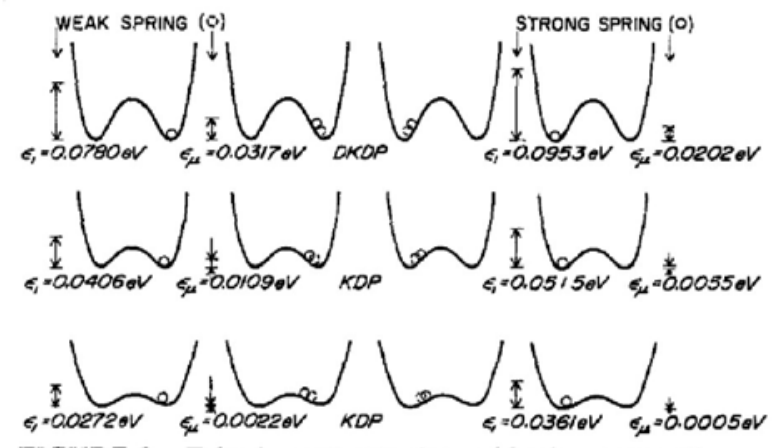

FIGURE 2 Takagi group energies and hydrogen positions for the even configuration for various well parameters and coupling strengths. Some dashed circles are omitted for ciarity.

that both DKDP and KDP have the same actual potential $U_{0}(x)=B_{0} x^{4}-A_{0} x^{2}$, and that

$$
U(x)=\int_{-\infty}^{\infty} \exp \left[-(y-x)^{2} / 2 y_{0}{ }^{2}\right] U_{0}(y) d y,
$$

with $y_{O D}$ and $y_{O H}$ found from deuteron and proton wavefunctions for a harmonic potential having the same curvature as at the minima of $U_{0}(x)$. The effective potentials $U(x)$ have $B=B_{0}$ but have $A_{0}$ replaced by $A_{D}$ for DKDP and by $A_{H}$ for KDP. The wells on the bottom line of Figure 2 have $A=\frac{1}{2} A_{H}$ and $B=\frac{1}{2} B_{0}$.

The values for $k^{\prime}$ are based on deuteron spin-lattice relaxation results in DKDP, which yielded $\epsilon_{1}$ of about $0.078 \mathrm{eV}\left(\epsilon_{1} / k=900 \pm 200 \mathrm{~K}\right) .{ }^{1}$ The values of $k^{\prime}$ chosen are for "weak springs" giving $\epsilon_{1} / k=900 \mathrm{~K}$ and "strong springs" giving $\epsilon_{1} / k=1100 \mathrm{~K}$. 


\section{TAKAGI GROUP CONCENTRATION AND MEAN FREE PATH}

The fractional Takagi group concentration obtained by minimizing the free energy is $N_{T} N=[1+$ $\left.e^{\left(\epsilon_{1}-\epsilon_{\mu}\right) / k T}\right]^{-1}$, so the average separation between Takagi groups is $d=N / N_{T} \geqslant 2$. For Takagi group length $n>2$ the Takagi groups would merge at high temperature, but no experimental evidence for such merging has been found in KDP-type crystals. Here $n$ is defined by $n=1+\sum_{i}\left|\left(x_{0}-x_{i}\right) / x_{0}\right|$ over the hydrogen positions in the even configurations.

The mean free path cannot exceed the mean Takagi group spatial separation $d a$, where $a=5.27 \AA$ is the interbond spacing. Scattering by phonons which see the Takagi group as a region with slightly higher (or lower) density has been calculated to have no significant effect. A third path-limiting process is thermalization by interactions with neighboring heavy atoms. The standard assumption is a probability $v_{0} e^{-u}$ per unit time for hops to adjacent sites, where $u=\epsilon_{\mu} / k T$. If immediately after hopping the Takagi group has probability $e^{-u}$ of making another hop in the same direction instead of stopping, its mean free path is $\lambda=a /\left(1-e^{-u}\right)$.

\section{DOMAIN WALL MOBILITY}

A domain wall is an unpolarized layer in which the preceding expression for $N_{T} / N$ is approximately correct. ${ }^{1}$ With a field present, the barrier heights become $\epsilon_{\mu} \pm \frac{1}{2} p E$ which substituted in the hoppinglimited expression for $\lambda$ yields Takagi group drift velocity $v_{d}=a v_{0}(p E / k T) e^{-u} /\left(1-e^{-u}\right)^{2}$, where $p$ has values $2.44 \times 10^{-28}$ and $3.03 \times 10^{-28}$ coul-cm for $\mathrm{KDP}$ and DKDP respectively. For $\nu_{0}=k T / h$ and $\mu_{w}=v_{d} N_{T} / N E$ the mobilities $\mu_{w}$ shown in Table I for temperatures of $100 \mathrm{~K}$ in $\mathrm{KDP}$ and $200 \mathrm{~K}$ in DKDP are obtained. The half-strength potential yields a strong isotope effect, but not as great as the measured $^{3}$ ratio of about $10^{6}$. The DKDP values agree quite well with previously reported results. ${ }^{1}$
The dielectric relaxation is assumed to be governed by a single relaxation time $\tau=P_{\infty} /(d P / d t)_{0}$, where $P_{\infty}=\epsilon_{0} \epsilon_{h} E$ (MKS units) and $(d P / d t)_{0}$ is evaluated when the small field $E$ is first applied. For $\lambda$ limited by annihilation with neighboring Takagi groups $\tau_{a}=h \epsilon_{0} \epsilon_{h} / N p^{2} e^{-u}$, while for hopping-limited $\lambda$, $\tau_{h}=\tau_{a}\left(1-e^{-u}\right)^{2} N / N_{T}$. Assuming $\tau=\tau_{a}+\tau_{h}$ and using 28 and 40 for the H-bond-dependent part $\epsilon_{h}$ of the dielectric constants for KDP and DKDP respectively at $300 \mathrm{~K}$, the values for $\tau$ given in Table I are obtained. Deuteration increases $\tau$ by roughly a factor of 10. The DKDP values are in general agreement with previous calculated and measured results. ${ }^{1}$

\section{DISCUSSION}

By introducing the concept of an extended Takagi group and decomposing the energies of such groups into creation and mobility components, it is possible to predict quite strong isotope effects in KDP without invoking cooperative tunneling. An isotope effect on Takagi group energies was obtained by taking account of quantum-mechanical effects of the isotope mass differences in a qualitatively reasonable way. Isotope effects on the Slater group energies could be found in a similar manner for a two- or three-dimensional model.

The above-described cooperative motion involves only the hydrogens in an extended Takagi group. Coupling of such motion with a heavy-ion TO mode to obtain a coupled "soft mode" is now being studied.

\section{REFERENCES}

1. V. H. Schmidt, Phys. Rev. 164, 749 (1967) and 173, 630 (1968) and references therein. Because of an error of a factor of 2 in the theory on page 754, the pre-exponential times $T_{0}$ on that page and in Figure 5 should be half as large as given.

2. Recent results of R. J. Nelmes, V. R. Eirikson and K. D. Rousc, Solid State Commun. 11, 1261 (1972) are $x_{0}=$ 0.22 and $0.17 \AA$ for DKDP and KDP respectively.

3. J. L. Bjorkstam and R. E. Oettel, Proc. Internat. Meeting on Ferroelectricity, Prague, 1966, Vol. II, page 91.

TABLE I

Takagi group length $n$, domain wall mobility $\mu_{w}$, and dielectric relaxation time $\tau$ for various well parameters and coupling strengths.

\begin{tabular}{llllllr}
\hline Xtal & Coupling & $A$ & $B$ & $n$ & $\begin{array}{l}\mu_{w}, \mathrm{~cm}^{2} / \mathrm{V}-\mathrm{sec} \\
\left(T_{c}-22 \mathrm{~K}\right)\end{array}$ & \multicolumn{1}{c}{$\begin{array}{r}\tau, \mathrm{sec} \\
(300 \mathrm{~K})\end{array}$} \\
\hline DKDP & weak & $A_{\mathrm{D}}$ & $B_{0}$ & 1.26 & $3.54 \times 10^{-4}$ & $19.1 \times 10^{-12}$ \\
KDP & weak & $A_{\mathrm{H}}$ & $B_{0}$ & 1.39 & $3.26 \times 10^{-4}$ & $3.1 \times 10^{-12}$ \\
KDP & weak & $\frac{1}{2} A_{\mathrm{H}}$ & $\frac{1}{2} B_{0}$ & 1.79 & $1.54 \times 10^{-2}$ & $1.5 \times 10^{-12}$ \\
DKDP & strong & $A_{\mathrm{D}}$ & $B_{0}$ & 1.47 & $1.98 \times 10^{-4}$ & $18.2 \times 10^{-12}$ \\
KDP & strong & $A_{\mathrm{H}}$ & $B_{0}$ & 1.70 & $2.16 \times 10^{-4}$ & $2.1 \times 10^{-12}$ \\
KDP & strong & $\frac{1}{2} A_{\mathrm{H}}$ & $\frac{1}{2} B_{0}$ & 2.32 & $9.00 \times 10^{-2}$ & $1.4 \times 10^{-12}$ \\
\hline
\end{tabular}

